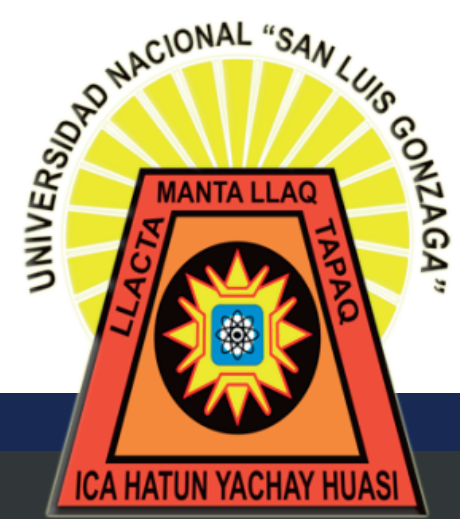

p-ISSN 2223-2893

e-ISSN 2225-6989

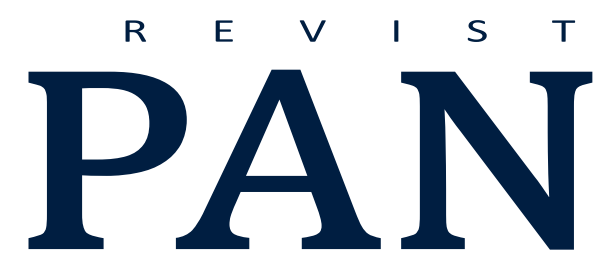

UNIVERSIDAD NACIONAL SAN LUIS GONZAGA. ICA, PERÚ
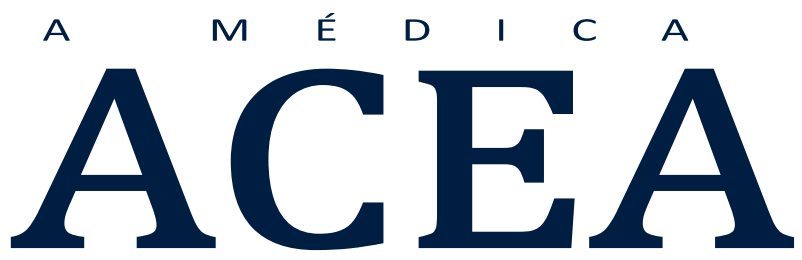

UNIVERSIDAD NACIONAL SAN IUIS GONZAGA.ICA, PERU

FACULTAD DE MEDICINA HUMANA "DANIEL ALCIDES CARRIÓN"

\title{
ARTÍCULO ORIGINAL:
}

VOLUMEN 10 NÚMERO 1 PUBLICACION CUATRIMESTRAL ENERO - ABRIL

\section{ACTIVIDAD FÍSICA COMO PREVENCIÓN DE SOBREPESO Y OBESIDAD EN NIÑOS DE 7-12 AÑOS.}

PHYSICAL ACTIVITY AS PREVENTION OF OVERWEIGHT AND OBESITY IN CHILDREN AGED 7-12 YEARS.

\section{AUTORES:}

DIANA MAYERLY VEGA VALDIVIESO

KAREN YULIANNA AMAYA PALACIOS

CARENTH JINNETH LINEROS FLOREZ

GRACIELA OLARTE RUEDA

INDEXADA EN:

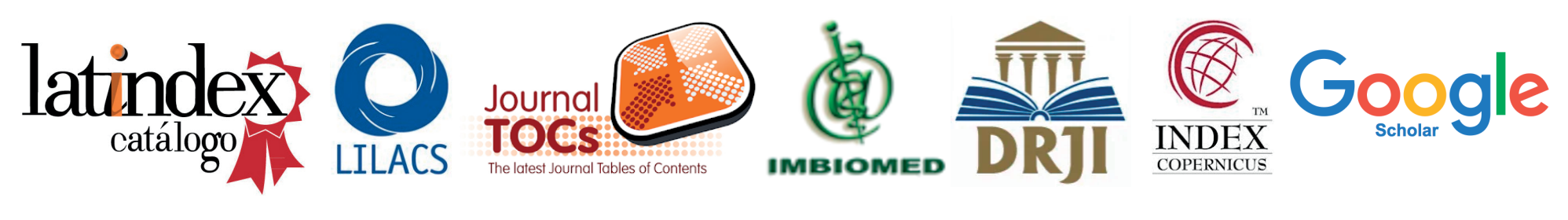

REVISTAS.UNICA.EDU.PE 


\section{ACTIVIDAD FÍSICA COMO PREVENCIÓN DE SOBREPESO Y OBESIDAD EN NIÑOS DE 7-12 AÑOS. \\ PHYSICAL ACTIVITY AS PREVENTION OF OVERWEIGHT AND OBESITY IN CHILDREN AGED 7-12 YEARS.}

Vega-Valdivieso Diana Mayerly', Amaya-Palacios Karen Yulianna², Lineros-Florez Carenth Jinneth ${ }^{3}$, Olarte-Rueda Graciela4.

1. Enfermera en formación, Fundación universitaria de San Gil, UNISANGIL. Orcid. https://orcid.org/0000-0003-0890-6328.

2. Enfermera en formación, Fundación universitaria de San Gil, UNISANGIL. Orcid: https://orcid.org/0000-0001-7637-6475.
3. Enfermera en formación, Fundación universitaria de San Gil, UNISANGIL. Orcid: https://orcid.org/0000-0002-0312-5322.

4. Enfermera, Magister en ciencias de la enfermería, especialista en epidemiología. San Gil -Santander

Orcid. https://orcid.org/0000-0003-1733-3285.

DOI: 10.35563/rmp.v10i1.402

\section{Correspondencia:}

Graciela Olarte Rueda

Dirección: Km 2 vía San Gil -

Charalá

Correo:

grueda@unisangil.edu.co

Celular: 3186258743

Correo electrónico:

grueda@unisangil.edu.co

Contribuciones de autoría: DVV, KAP, CLF y GOR: participaron en el diseño del estudio, análisis, redacción revisión y aprobación final del manuscrito.

Conflicto de intereses: no existen conflictos de intereses del autor o autores de orden económico, institucional, laboral o personal.

\section{Financiamiento:}

Autofinanciado.

\section{Cómo citar:}

Vega-Valdivieso Diana $M$, Amaya-Palacios Karen $Y$, Lineros-Florez Carenth J, Olarte-Rueda Graciela. Actividad física como prevención de sobrepeso y obesidad en niños de 7-12 años. Rev méd panacea. 2020;10(1) 32-37. DOI: 10.35563/rmp.v10i1.402

\section{RESUMEN}

Introducción: La obesidad y el sobrepeso son un problema de salud que en la actualidad está afectando en gran medida a los niños y niñas a nivel mundial y nacional. Objetivo: determinar el nivel de actividad física que maneja la población escolar de 7 a 12 años en dos colegios del área rural y urbana de municipio de San Gil, para la prevención del sobrepeso y la obesidad. Materiales y métodos: Estudio cuantitativo descriptivo de corte transversal, la muestra estuvo conformada por 102 estudiantes de dos instituciones publica, pata la recolección de los datos se utilizó el instrumento para valoración del estado nutricional (sobrepeso y obesidad) por medidas antropométricas y hábitos alimentarios, tomado de una investigación de la universidad de Cartagena, se aplicó estadística descriptiva. Resultados: Se encontró que el 33,3\% de los niños respondieron que hicieron actividad física 2 o 3 veces por semana, el 27,5\% la realizaron 4 a 5 veces por semana, el 17,5\% 6 a 7 veces por semana, 11,8\% no realizaron ninguna actividad física y 9,8\% 1 vez la última semana. Conclusiones: Se identificó que los escolares realizan activada física durante la semana, se encontraron casos de sobrepeso y obesidad.

Palabras clave: aumento de peso, estatura, factores de riesgo,(Decs).

\section{ABSTRACT}

Introduction: Obesity and overweight are a health problem that is currently affecting boys and girls to a great extent worldwide and nationally. Objective: to determine the level of physical activity handled by the school population aged 7 to 12 in two schools in the rural and urban areas of the municipality of San Gil, for the prevention of overweight and obesity. Materials and methods: Cross-sectional descriptive quantitative study, the sample was made up of 102 students from two public institutions, for data collection the instrument was used to assess nutritional status (overweight and obesity) by anthropometric measures and eating habits. taken from an investigation of the University of Cartagena, descriptive statistics were applied. Results: It was found that $33.3 \%$ of the children responded that they did physical activity 2 or 3 times a week, $27.5 \%$ did it 4 to 5 times a week, $17.5 \% 6$ to 7 times a week, $11.8 \%$ did not perform any physical activity and $9.8 \% 1$ time in the last week. Conclusions: It was identified that schoolchildren perform physical activity during the week, cases of overweight and obesity were found.

Keywords: weight gain, height, risk factors, (DESH). 


\section{INTRODUCCIÓN}

La obesidad y el sobrepeso son un problema de salud que actualmente afecta a muchas personas, pero en los últimos años se ha visto un incremento en los niños y niñas. Estos problemas de salud y las enfermedades conexas son en gran medida prevenibles. (1) Para la Organización mundial de la salud (2) se define la obesidad como "una acumulación anormal de grasa que puede ser perjudicial para la salud", a manera más fácil de identificar el exceso de peso es mediante el índice de masa corporal," que es el resultado de dividir el peso en kilogramos por la talla en metros al cuadrado $(\mathrm{kg} / \mathrm{m} 2)$. (3)

El sobrepeso y la obesidad Según la Encuesta Nacional de Situación Nutricional de Colombia (ENSIN)(4) 2015, en el rango de edad de 5-12 años "se incrementó de 18,8\% en 2010 a 24,4\% en 2015" y en el departamento de Santander "24 de cada 100 niños de 5 a 12 años presentan exceso de peso" (5). Así mismo entre las 6 - 11 años en el municipio de San Gil, 2009 - 2014, la obesidad tuvo una proporción de 5,8 \% (6).

Existen diversos factores de riesgo para presentar sobrepeso y obesidad tales como la alimentación, la actividad física, hábitos de sueño y el estrés son los hábitos más influyentes que predisponen principalmente a los niños y niñas,(7) se ha visto que actualmente los niños y las niñas han dejado de lado la actividad física por actividades muy sedentarias como la televisión, los videos juegos y el internet, lo que hace que se aumenta la preocupación por las consecuencias que esto pueda tener en la salud.

El incremento de la obesidad infantil se ve acompañado de complicaciones que implican riesgos futuros en la salud y la calidad de vida en los escolares entre los 5 a 12 años de edad, las complicaciones más frecuentes que sufre esta población es el "síndrome metabólico, presión arterial alta, ateroesclerosis, enfermedad del corazón, diabetes, niveles elevados de colesterol en sangre, distintos tipos de cáncer y trastornos del sueño entre otros". (8 - 9)

Por lo tanto, es importante el fomento de la actividad física como una de las estrategias para la reducción de estos problemas de salud, dado que tiene beneficios que contribuyen al bienestar de las personas, muchos estudios muestran que el deporte y actividad física son buenos para la salud y les permiten sentirse más a gusto consigo mismos; es decir, que afecta positivamente al bienestar integral del individuo. (10-11).

En el año 2013 -2014 en "Santander el 27,0\% de la población de 5 a 17 años se clasificó en sobrepeso y obesidad según el indicador del índice de masa corporal (IMC) para la edad".(12) Según sexo, se encontró que el" sobrepeso fue mayor en mujeres, mientras que la obesidad fue superior en hombres"(13); actualmente en el municipio de San Gil no hay evidencias de estudios realizados con relación a factores predisponentes y tampoco estrategias que hayan dado resultados positivos en cuanto a la disminución de los índices de esta problemática.

Por lo anterior se ve la necesidad de reintroducir la actividad física a los niños y niñas, con el fin de prevenir enfermedades que en la edad adulta puedan afectar su calidad de vida, esto se puede lograr a través de la creación de una estrategia educativa para la disminución del sobrepeso y la obesidad enfocada en la población escolar de 7 a 12 años de dos colegios del municipio de San Gil. Según el Consejo de ministros de salud de Centroamérica y República Dominicana (14) "la estrategia debe estar enfocada en los grupos de niños en edad preescolar, escolar y adolescente, ya que la evidencia demuestra que entre más temprano un individuo presenta sobrepeso u obesidad, mayor es su riesgo de mantenerse con sobrepeso u obesidad en los años posteriores y mayor la probabilidad de sufrir morbilidades relacionadas a dichas condiciones de salud". Así mismo estos problemas generar consecuencias emocionales y sociales. (15)

De ahí la importancia de evaluar el nivel de la actividad física en los niños escolares de 7 a 12 años para medir su relación con el sobrepeso y la obesidad, y de esta manera desde el enfoque de enfermería diseñar estrategias que ayuden a la prevención y a la promoción de la salud en niños y niñas del Municipio de San Gil.

La finalidad del estudio es determina el nivel de actividad física que maneja la población escolar de 7 - 12 años en 2 colegios del área rural y urbana del municipio de San Gil, para la prevención del sobrepeso y la obesidad.

\section{MATERIALES Y MÉTODOS}

Se realizó un estudio descriptivo de corte transversal la muestra estuvo conformado 102 niños y niñas, la información se recolectó a través del cuestionario de actividad física para niños mayores PAQ-C. Consta de 9 ítems que averiguan él tipo de actividad física en el tiempo libre, la frecuencia con la que se realizó actividad durante la semana, en clase de educación física, después de la escuela, en horas de la tarde, y en el fin de semana.

Las variables de análisis fueron género, edad, peso, talla, IMC, actividad física, deportes juegos y aeróbicos, donde se describieron en frecuencias y porcentajes.

El procedimiento para la recolección de los datos se estructuró en cinco etapas; la primera fue la solicitud y permisos para la aplicación del instrumento. En la segunda etapa se solicitó la autorización para firmar el consentimiento informado a los padres de familia o responsables de los niños y niñas, una vez firmado el consentimiento se procedió a la aplicación de encuesta y la toma de medidas antropométricas a cada uno de los niños y niñas, se utilizó la báscula y tallímetro en una superficie plana horizontal y firme. Con un posterior análisis de los datos por medio de Microsoft Excel versión 2013, se procesó la información en el programa estadístico SPSS versión 24 por último, el diseño de una estrategia de prevención del sobrepeso y la obesidad basada en un plan de alimentación y ejercicios.

De acuerdo con la Resolución 8430 de 1993, este estudio corresponde a una investigación sin riesgo. Conto con la respectiva aprobación del comité ético de la fundación universitaria UNISANGIL en acta número 005-18.

\section{RESULTADOS}

\section{Datos antropométricos}

Respecto al peso para la talla se encontró que el $25,5 \%$ de los niños están con peso igual a la media (percentil 50), el 19,6\% con un peso ligeramente por debajo de la media (percentil 25), el $18,6 \%$ con peso superior a la media (percentil 90 ) y el 11,8 estaba en el límite alto de peso (percentil 97), así mismo se encontró que el 38,2\% tenían un IMC normal, el 20,6\% en sobrepeso y el $6,8 \%$, en obesidad. 


\section{Actividad física}

Por otra parte, se encontró que el 34,3\% caminaron como ejercicio 1 o 2 veces por semana, el 26,5\% saltaron o corrieron 3 a 4 veces por semana, el 16,7\% montaron bicicleta, saltaron y corrieron 5 a 6 veces por semana y el 18,6\% de los niños saltaron o corrieron 7 o más veces por semana.

Se logró identificar que los días que más realizan actividad física son el día viernes con un $11,8 \%$, el domingo $9,8 \%$, el sábado $8,8 \%$, el miércoles $6,9 \%$, el lunes y martes $2,9 \%$ y el jueves $1,0 \%$. Estos resultados están muy asociados a los días en los que tiene la clase de educación física.

En los últimos 7 días, el $66,7 \%$ de los niños y niñas antes y después del almuerzo se sentaron (hablando, leyendo, haciendo trabajos escolares), el $13,7 \%$ de los niños jugaron un poco, el $11,8 \%$ se mantuvieron parados o caminando por los alrededores, el 6,9\% corrieron o jugaron bastante y el 1,0\% corrieron o jugaron fuerte mucho tiempo.

En cuanto a si tuvieron alguna complicación de salud que les impidió realizar actividad física se encontró que el 91,2\% no presentó ningún problema de salud y el $8,8 \%$ si tuvo alguna afección en su salud.

De acuerdo al último fin semana se encontró que el 38,2\% de los niños realizaron actividad física 2 o 3 veces el último fin de semana, el $24,5 \%$ una vez, el $16,7 \% 4$ a 5 veces, el 10,8\% 6 o más veces y el 9,8\% no realizó ninguna actividad física el último fin de semana.

\section{DISCUSIÓN}

El fomento de la actividad física es uno de los aspectos más importantes en la prevención de sobrepeso y obesidad por lo tanto el objetivo del presente estudio fue determinar el nivel de actividad física de los niños y niñas de 7 a 12 años con el fin de prevenir el sobrepeso y la obesidad.

Se encontró que el 38,2\% tenían un IMC normal, el 34,3\% en bajo peso, el $20,6 \%$ en sobrepeso y el $6,8 \%$, en obesidad, comparado con el estudio de Acosta Favela, et al. (16) La relación Índice de Masa Corporal-sexo, nos indica que del 100\% de participantes evaluados el $30 \%$ de niños y el $27 \%$ de niñas presentan un peso normal, el $5 \%$ de niños y el $8 \%$ de niñas presentan sobrepeso y solo $1 \%$ en niños presentan obesidad. Por otra parte, el $18 \%$ en niños y el $11 \%$ niñas presentan desnutrición. En el estudio de Reyes-Sepeda, J. (17) Se encontraron datos muy similares de la prevalencia total de obesidad y sobrepeso en niños fue de $37.8 \%$, es decir, 46 casos, (14.8\%) niños se clasificaron con sobrepeso y 28 (23\%) con obesidad. Estos resultados muestran que, si existe sobrepeso y obesidad, aunque no son porcentajes muy altos, son un problema de salud que se debe intervenir, para prevenir enfermedades crónicas a futuro.

Una de las formas de prevenir es a través de estrategias que ayuden a disminuir estas enfermedades que pueden afectar la salud de los niños y niñas de la población, según Aguilar (18) es un estudio: Obesidad en el niño: Factores de riesgo y estrategias para su prevención, manifiesta:

Es urgente la implementación de intervenciones educativas que contribuyan a disminuir el exceso de peso de la población peruana, con énfasis en niños y adolescentes, a través de la promoción de los estilos de vida saludables en el núcleo familiar, así como el consumo de alimentos saludables, mayor actividad física con disminución de actividades sedentarias y menor número de horas de uso de medios audiovisuales y adecuadas horas de sueño en la población en general.
Estas estrategias deben de ser aplicadas en los colegios donde los niños pasan la mayor parte de tiempo".

Según la organización mundial de la salud OMS (19) "Los niños y jóvenes de 5 a 17 años inviertan como mínimo 60 minutos diarios en actividades físicas de intensidad moderada a vigorosa". En el estudio la actividad física que realizaron los niños y niñas en los último siete días de la semana fue del 39,2\% respondió que a menudo ( 3 o 4 veces la semana pasada), el $26,5 \%$ respondieron bastante a menudo ( 5 o 6 veces la semana pasada) y el $20,6 \%$ a veces (1o 2 veces la semana pasada).

Dentro de la actividad que realizaron en su tiempo libre caminaron como ejercicio 1 o 2 veces por semana, saltaron o corrieron 3 a 4 veces por semana, montaron bicicleta y saltaron y corrieron 5 a 6 veces por semana y saltaron o corrieron 7 o más veces por semana. Esto coincide con la organización mundial de la salud (20) la cual manifiesta que, para los niños y jóvenes de este grupo de edades, la actividad física consiste en juegos, deportes, desplazamientos, actividades recreativas, educación física o ejercicios programados, en el contexto de la familia, la escuela o las actividades comunitarias.

En el estudio se encontró que durante la clase de educación física los niños y niñas siempre estuvieron muy activos. Según Abarca (21) "El centro escolar representa una oportunidad para promocionar la actividad física, porque los niños permanecen gran parte de su tiempo en él." Resultando que para obtener "un mayor provecho en los entornos escolares en la promoción de la actividad física se debe reconocer la creencia y las actitudes de los estudiantes hace el movimiento del cuerpo humano ". (22) Así mismo se debe tener en cuenta en el diseño de estas estrategias eficaces que además se debe trabajar temas como la autoestima, la resolución de conflictos y el ambiente familiar. (23)

De acuerdo al tiempo en horas que pasan los niños y niñas al día frente a la pantalla de un computador fue de 2 a 6 horas, en el televisor de 2 a 4 horas y en videojuegos de 6 a 10 horas, así mismo en el estudio de Lois y Rial: Hábitos alimentarios y de actividad física de alumnado de Educación Primaria, se encontró que el $41,33 \%$ de alumnos ven la televisión dos horas al día y que $25,33 \%$ de alumnas ven la televisión 4 horas al día, siendo un cómputo bastante elevado.(24) Según la encuesta nacional de la situación nutricional en Colombia "el tiempo excesivo frente a pantallas, aquel dedicado a actividades sedentarias como ver televisión o jugar con videojuegos, afecta a siete de cada diez escolares de áreas urbanas, frente a cinco de cada diez de zonas rurales; el problema es más marcado entre la población de mayores ingresos, afectando a ocho de cada diez menores".(25) En diferentes estudios se ha demostrado que el uso excesivo de la televisión y jugar videojuegos están asociados al sobrepeso y a la obesidad. (26)

En los resultados anteriormente mencionados se puede evidenciar que el nivel de sedentarismo que están presentando los niños y niñas de la educación primaria, algo que preocupa teniendo en cuenta que esta conducta se relaciona con un mayor riesgo cardiovascular. (27) Todo esto por el agravante de las nuevas tecnologías y un aumento del abandono de la práctica deportiva. (28) De igual forma los niveles inferiores de aptitud física conlleva a que interactúen menos con su entorno. (29) $Y$ esto puede tener un "impacto negativo en su desarrollo conduciéndoles a niveles de habilidades motoras muy pobres. (30) 
Es una problemática que día a día está siendo más evidente debido a todos los cambios en los patrones socioculturales de la comunidad, así como el acceso a las tecnologías las cuales están influyendo en la inactividad física de los niños y niñas; por otro parte existen otros factores de riesgo que favorecen la aparición de sobrepeso y obesidad.

\section{CONCLUSIONES}

De acuerdo al índice de masa corporal los niños y las niñas se encuentran en un índice normal, seguido de sobrepeso y finalmente un porcentaje menor en obesidad.

Los niños y niñas realizaron activada física en su mayoría de 3 a 4 veces por semana, dentro de las actividades que más practicaron fue caminar, salta, correr y montar bicicleta, así mismo se encontró que los niños realizaron actividad física 2 o 3 veces en el último fin de semana. 


\section{REFERENCIAS BIBLIOGRÁFICAS}

1. Organización Mundial de la Salud OMS. Sobrepeso y obesidad infantil. [Internet].2014; [consultado septiembre 12 de 2018] disponible en: http://www.who.int/dietphysicalactivity/childhood/es/.

2. Ibíd.

3. Anzar, L; Alonso M. protocolos diagnostico terapéuticos de gastroenterología, hepatología y nutrición pediátrica. Obesidad. [Internet]. 2018. [acceso mayo 13 de 2019]. disponible

en: https://www.aeped.es/sites/default/files/documentos/obesi dad.pdf

4. Encuesta Nacional de Situación Nutricional de Colombia ENSIN. [Internet] .2015; [acceso noviembre 11 de 2018]. Disponible

en: https://www.icbf.gov.co/bienestar/nutricion/encuesta-nacio nal-situacion-nutricional

5. Encuesta Nacional de Situación Nutricional de Colombia (ENSIN). [Internet] .2015; [acceso noviembre 11 de 2018]. Disponible en: https://www.minsalud.gov.co/Paginas/Bucaramanga-cerro-l anzamiento-de-Encuesta-Nacional-de-Situacion-Nutriciona I-de-Colombia-ENSIN-2015.aspx

6. Acuerdo municipal Nro. 028. Por medio del cual se aprueba y se adopta el plan de seguridad alimentaria y nutricional para el municipio de san gil 2014-2019". [Internet].2014. [acceso octubre 17 de 2018]. Disponible en: http://www.concejosangil.gov.co/documentos/Acuerdos/Ac uerdos\%202014/Acuerdo\%20028\%20de\%202014.pdf

7. Bodega P; Fajardo D; García B; García N, Garicano E, Megías A; Micó V; Rodríguez P; Romero, E. Influencia de hábitos saludables en el estado ponderal de niños y adolescentes en edad escolar. Nutrición hospitalaria. [Internet] 2015, [acceso octubre 17 de 2018].Disponible en: http://scielo.isciii.es/pdf/nh/v31n5/12originalobesidad04.pd $f$.

8. National Heart, Lung, and Blood Institute NIH (2015). Sobrepeso y obesidad. [Internet] 2015 [acceso octubre 17 de 2018]. Disponible en: https://www.nhlbi.nih.gov/health-topics/espanol/sobrepeso -y-obesidad.

9. Asociación colombiana de endocrinología. Obesidad infantil. [Internet]. 2017 [acceso octubre 20 de 2018]. Disponible en: https://www.minsalud.gov.co/Documentos\%20y\%20Publica ciones/Obesidad\%20infantil.pdf.

10. Ascencio J, Daza A; Jiménez M; Nájera Y; \& Suarez M. Estilos de vida saludable en adolescentes relacionados con alimentación y actividad física: una revisión integrativa. Revista salud. [internet]. 2016[acceso octubre 19 de 2018].Disponible en: http://publicaciones.unisimonbolivar.edu.co/rdigital/ojs/ind ex.php/saludmov/article/view/1619/1495

11. ibíd.

12. Clavijo S, Plata V. Situación nutricional y perfil metabólico de la población de 0 a 17 años en Santander: estudio SALUS. [Internet]. 2017 [acceso octubre 10 de 2018].Disponible en: http://observatorio.co/web/publicaciones/situacion_nutri_\% 20perfil_metabolico_estudio_SALUS.pdf

13. Bodega P; Fajardo D; García B; García N, Garicano E; Mauro I; Megías A. Micó V; Rodríguez P; Romero E. Influencia de hábitos saludables en el estado ponderal de niños y adolescentes en edad escolar. Nutrición hospitalaria. [Internet].2015) [acceso octubre 17 de 2018].Disponible en: http://scielo.isciii.es/pdf/nh/v31n5/12originalobesidad04.pdf
14. Consejo de ministros de salud de centro América y República Dominicana COMISA Estrategias para la prevención de sobrepeso y la obesidad en la niñez y la adolescencia de centro América y Republica dominicana. [Internet] .2014 [acceso septiembre 15 de 2018]. Disponible e $n$

http://copal.org.ar/wp-content/uploads/2015/06/201406-c omisca-estrategia_obesidad.pdf

15. Rodríguez P. Estrategias para prevenir la obesidad y sobrepeso en niños escolares. [Internet]. 2016 [acceso septiembre 5 de 2018]. Disponible en: https://uvadoc.uva.es/bitstream/handle/10324/21417/TFGL1453. pdf;jsessionid =17CD26C0C82DB932C15A7B7F4ED19 $\mathrm{D} 4 \mathrm{D}$ ? sequence $=1$

16. Acosta F, Medrano D; Duarte G, González R. Malos Hábitos Alimentarios y Falta de Actividad Física Principales Factores Desencadenantes de Sobrepeso y Obesidad en los Niños Escolares. [Internet] ;2017 [acceso octubre 11 de 2018].54 (11). Disponible en: http://148.210.132.19/ojs/index.php/culcyt/article/view/871 $/ 822$

17. Reyes J; García E, Gutiérrez J; Galeana J; Gutiérrez M. [Internet]2016 [Consultado Febrero de 2019].Disponible en: https://www.medigraphic.com/pdfs/sanmil/sm-2016/sm161 I.pdf

18. Aguilar C. Obesidad en el niño: Factores de riesgo y estrategias para su prevención en Perú. [Internet], 2017 [Consultado octubre 15 de 2018].34 (1). Disponible en: https://www.scielosp.org/scielo.php?script=sci_arttext\&pid =S1726-46342017000100113

19. Organización mundial de la salud. OMS. La actividad física en los jóvenes. [Internet] . 2018. [acceso noviembre 5 de 2018]. Disponible en: https://www.who.int/dietphysicalactivity/factsheet_young_p eople/es/

20. Ibid.

21. Abarca A; Murillo B; Clemente J; Zaragoza J; Generelo, E. La Educación Física: ¿Una oportunidad para la promoción de la actividad física? [Internet] .2015. [acceso Marzo de 2019]. Disponible en: https://zaguan.unizar.es/record/70302

22. Álvarez C. Entendiendo los factores que determinan la actividad física en el entorno escolar desde la perspectiva de los niños y niñas. [Internet]. 2016 [acceso marzo de 2019]. Disponible https://revistas.una.ac.cr/index.php/mhsalud/article/view/85 83/9813

23. Golpe Ferreiro; Isorna Manuel; Gómez P; Boubeta R. Uso problemático de Internet y adolescentes: el deporte sí importa. [Internet] .2017 [Consultado marzo 9 de 2019]. Disponible https://www.redalyc.org/pdf/3457/345750049010.pdf.

24. Lois L, Rial T. Hábitos alimentarios y de actividad física de alumnado de Educación Primaria: estudio descriptivo de un colegio de Pontevedra. [Internet] 2016[acceso o octubre 18 de 2018]. Disponible en: https://ruc.udc.es/dspace/bitstream/handle/2183/17708/SP ORTIS_2_1_2016_5.pdf? sequence=1\&isAllowed =y

25. Ariza M, Galeano L, Martínez E, Marllelys Villanueva $M$; Herazo Y. Sedentarismo en estudiantes de la escuela Instenalco. [Internet] 2015 [Consultado Noviembre de 2018]; disponible en: http://publicaciones.unisimonbolivar.edu.co/rdigital/ojs/ind ex.php/saludmov/article/view/1341/1324

26. Encuesta Nacional de Situación Nutricional. ENSIN 2015. [Internet] .2019 . [Consultado Mayo de 2019]; disponible en: 
https://www.icbf.gov.co/bienestar/nutricion/encuesta-nacio nal-situacion-nutricional

27. Díaz R; Aladro C. Relación entre uso de las nuevas tecnologías y sobrepeso infantil, como problema de salud pública. [Internet] 2016 [Consultado Abril de 2019]. Disponible en: file://C:/Users/UNISANGIL/Downloads/Dialnet-RelacionEnt reUsoDeLasNuevasTecnologiasYSobrepesoln-5353331.pdf

28. Castro S; Linares M; Sanromán S; Pérez A. Análisis de los comportamientos sedentarios, práctica de actividad física y uso de videojuegos en adolescentes. [Internet] .2017. [Consultado febrero de 2019]. Disponible en: https://ruc.udc.es/dspace/handle/2183/22766.

29. Cigarroa I; Carla S; Zapata R. Efectos del sedentarismo y obesidad en el desarrollo psicomotor en niños y niñas: Una revisión de la actualidad latinoamericana. [Internet] 2015. [Consultado Febrero 18 de 2019]; disponible en: https://www.researchgate.net/profile/lgor_Cigarroa/publica tion/309601231_Efectos_del_sedentarismo_y_obesidad_en_ el_desarrollo_psicomotor_en_ninos_y_ninas_Una_revision_d e_la_actualidad_latinoamericana/links/5840544f08ae61f75 d cee10b.pdf

30. Ibíd. 